\title{
Pinning of Tumoral Growth by Enhancement of the Immune Response
}

\author{
A. Brú, ${ }^{1,2, *}$ S. Albertos, ${ }^{3}$ J. A. López García-Asenjo, ${ }^{4}$ and I. Brú ${ }^{5}$ \\ ${ }^{1}$ CCMA, Consejo Superior de Investigaciones Científicas, Serrano 115, 28006 Madrid, Spain \\ ${ }^{2}$ Department Matemática Aplicada, Facultad de CC. Matemáticas, Universidad Complutense de Madrid, \\ Avenue Complutense, 28040 Madrid, Spain \\ ${ }^{3}$ Servicio de Aparato Digestivo, Hospital Clínico San Carlos, Avenue Martín Lagos, 28003 Madrid, Spain \\ ${ }^{4}$ Servicio de Anatomía Patológica, Hospital Clínico San Carlos, Avenue Martín Lagos, 28003 Madrid, Spain \\ ${ }^{5}$ Centro de Salud La Estación, Paseo del Muelle 70, 45600 Talavera de la Reina, Toledo, Spain
}

(Received 5 September 2003; published 7 June 2004)

Tumor growth is a surface phenomenon of the molecular beam epitaxy universality class in which diffusion at the surface is the determining factor. This Letter reports experiments performed in mice showing that these dynamics can, however, be changed. By stimulating the immune response, we induced strong neutrophilia around the tumor. The neutrophils hindered cell surface diffusion so much that they induced new dynamics compatible with the slower quenched-disorder Edwards-Wilkinson universality class. Important clinical effects were also seen, including remarkably high tumor necrosis (around $80 \%-90 \%$ of the tumor), a general increase in survival time [the death ratio in the control group is 15.76 times higher than in the treated group (equivalent to a Cox's model hazard ratio of $0.85 ; 95 \%$ confidence interval $0.76-0.95, p=0.004)$ ], and even the total elimination of some tumors.

DOI: $10.1103 /$ PhysRevLett.92.238101

The recent discovery of the universality of tumor dynamics [1,2] contradicts the "standard model" of tumor growth, and has important implications for the treatment of cancer. These dynamics, which are the same for tumors of different types, from different organs, with different phenotypes or genotypes, and both in vivo or in vitro, all involve a surface growth phenomenon classifying them within the molecular beam epitaxy (MBE) universality class. The reason for such classification lies in an effect which has hitherto passed unnoticed [1]: Cell proliferation takes place almost exclusively at the border of the tumor, and newborn cells diffuse along the tumor interface with healthy tissue before they settle at a fixed position. This diffusion tends to place new cells in concavities [1], where they "maximize the number of neighbors"- a microscopic mechanism known to be responsible for MBE growth [3,4].

It is well established that it is at the convexities of the tumor interface where cells can achieve a better supply of nutrients and oxygen [2]. In addition, the $p \mathrm{H}$ at these positions is more favorable (less acidic): A consequence of the large number of tumor cells at the concavities is the high concentration of lactic acid produced by their metabolism. This led to the belief that tumors grow preferentially at the convexities. However, our experiments indicate that cells move preferentially towards the "unfavorable" concavities [1,2]. In the cell lines and tumor types that we have grown in vitro, we have even observed this cell diffusion [2] (its existence in tumors in vivo is inferred from the fact that the exponents which characterize the dynamics of growth are the same as those found in vitro).

To explain such "illogical" behavior requires there to be some compensation for the loss of supplies and settling
PACS numbers: 87.10.+e, 02.50.Ey, 05.45.Df, 68.35.Fx

in a more hostile environment. This is found in the cells gaining more room and therefore experiencing less pressure. The host tissue and the immune response exert a pressure on tumors which opposes their growth, but by sitting at the concavities of the interface the cells keep this pressure to a minimum. This is consistent with the observation that tumor interfaces are always super-rough [1], a very favorable pattern for withstanding pressure. Accordingly, tumors must first destroy the host tissue to be able to invade it and occupy the new free space. This is in contrast to the common belief that tumors first invade the host tissue and later destroy it.

To explore this new concept of cancer growth, and to analyze the effect of pressure at the tumor border, we increased the immune response to see whether these universal growth dynamics could be modified. The immediate effect was the massive movement of neutrophils towards, and eventually their surrounding of, the tumor. Neutrophils participate in all stages of the immune response and are the first cells to arrive at a target area. They are therefore the first emergency barrier of the body [5]. Formed in the bone narrow, they enter the bloodstream, and from there pass into the affected tissues. They are very massive cells and are resistant to the acidic $p \mathrm{H}$ of tumor concavities.

Ehrlich tumors were implanted subcutaneously into the thigh muscle of $20 \mathrm{C} 57 \mathrm{BL} / 6$ mice. These tumors were initially formed from a single-cell suspension containing cells of the fibroehrlich cell line (a type of tumor fibroblasts). When the mice developed a palpable subcutaneous neoplastic mass, a daily dose of $10 \mu \mathrm{g} / \mathrm{kg} /$ day of granulocyte-macrophage colony stimulating factor (GM-CSF) was injected into 16 of them in order to increase their immune response. The remaining four mice formed the 
control group. After 15 days of therapy, six of the 16 treated mice were sacrificed to analyze their tumors. In the remainder, the treatment was prolonged for two months (or until death). The dose of GM-CSF remained the same throughout the course of treatment.

During treatment, the tumors were monitored by nuclear magnetic resonance (NMR) imaging to analyze the extent of necrosis. Postcontrast T1-weighted images were obtained both in the sagittal and coronal planes in six mice (five treated mice and one control mouse). The animals received a solution of gadolinium before NMR to highlight active tumor areas. They were then anesthetized with isoflurane (3\% for induction and $1 \%-1.5 \%$ for maintenance) in medical oxygen $(2 \mathrm{l} / \mathrm{min}$ for induction and $1 \mathrm{l} / \mathrm{min}$ for maintenance) and NMR was performed using a BIOSPEC BMT 47/40 (Bruker, Ettlingen, Germany) equipped with a $12 \mathrm{~cm}$ actively shielded gradient system, operating at $4.7 \mathrm{~T}$. All animals were held still in the prone position using a cylindrical cage with an inner diameter of $4 \mathrm{~cm}$ and a length of $6 \mathrm{~cm}$. Global shimming was performed followed by three scout images in the axial, sagittal, and coronal planes. For all animals, coronal and sagittal $\mathrm{T} 1$ weighted spin-echo $(T R / T E=700 / 15 \mathrm{~ms}$, where TR refers to repetition time and TE to echo time) scans were performed. The matrix size was $256 \times 256$. The field of view (FOV) used was $4 \mathrm{~cm}$ and the slice thickness 1.5 or $2.0 \mathrm{~mm}$. The number of slices taken varied between 15 and 26 depending on tumor size. In some cases, axial T1 weighted scans were also performed using the same parameters. For control animals, T2 weighted fast spin-echo $(T R / T E=$ $2000 / 80 \mathrm{~ms}$ ) images were acquired using the same geometrical parameters as above. After evaluating the degree of necrosis, histological specimens (see below) were correlated with these images.

After the death of the mice, histological sections $(4 \mathrm{~mm})$ of their tumors were analyzed using paraffinembedded material on poly-L-lysine-coated glass slides. After deparaffinating and rehydration, the sections underwent three rounds of $3 \mathrm{~min}$ each of autoclave treatment in antigen retrieval solution (DAKO). Endogenous peroxidase activity was then blocked with hydrogen peroxide for $15 \mathrm{~min}$. Sections were then incubated with biotinylated secondary antibody for $20 \mathrm{~min}$ followed by treatment with streptavidin-biotin-peroxidase complex (LSAB kit, DAKO) for another $20 \mathrm{~min}$ at room temperature. Sections were rinsed between steps with several changes of phosphate-buffered saline. Color was developed with diaminobenzidine tetrahydrochloride. Sections were lightly counterstained with hematoxylineosin. The sections were photographed under the light microscope (Canon; magnification $100 \times$ with a final resolution of $2.62 \mu \mathrm{m} /$ pixel.

Photographs of the same tumor were loaded onto a computer and the tumor profiles hand traced. Scaling analysis and other measurements were performed on these profiles using in-house computer software. The experiment was designed to detect an absolute difference in tumor decline in the GM-CSF-treated group. Efficiency analyses included all mice that received treatment. The safety and efficiency of treatment was assessed at the end of treatment. Statistical analysis involved studying the event distribution over time (Breslow's test) [6,7] and estimating the risk of death using Cox's model of proportional risks (hazard ratio and $95 \%$ of CI) [8].

In all cases, for all mice, tumor contours were analyzed and the corresponding local and global roughness exponents calculated. These exponents arise from the power law behavior of the interface width (a measure of local fluctuations of the interface at about its local average value) which follows:

$$
w(l, t)= \begin{cases}l^{\alpha_{\mathrm{loc}}} & \text { if } l<L \\ l^{\alpha_{\mathrm{glob}}} & \text { if } l=L,\end{cases}
$$

with $l$ being the arclength and $L$ the whole contour length of the circular interface [1]. $\alpha_{\text {loc }}$ and $\alpha_{\text {glob }}$ are the local and global roughness critical exponents, respectively. Figure 1 shows the power spectrum of the tumor (the Fourier transformation of the tumor contour) of a mouse from the control group. From the slope $\left(=-2 \alpha_{\text {glob }}-1\right)$ is obtained for the global roughness exponent, $\alpha_{\text {glob }}^{\text {Control }}=$ $1.50 \pm 00.10$, a value fully compatible with MBE dynamics $[1-4,9,10]$. MBE is described by a Langevin-like equation with a fourth order derivative term,

$$
\frac{\partial}{\partial t} h(x, t)=\frac{\partial^{4}}{\partial x^{4}} h(x, t)+F+\eta(x, t)
$$

with $h(x, t)$ describing the position of the interface, $F$ being a (constant) driving force and representing a cell division rate, and $\eta(x, t)$ the thermal noise with a mean of zero and correlations $\left\langle\eta(x, t) \eta\left(x^{\prime}, t^{\prime}\right)\right\rangle=$ $2 D \delta\left(x-x^{\prime}\right) \delta\left(t-t^{\prime}\right)$. The theoretical global and local roughness critical exponents corresponding to the MBE universality class are $\alpha_{\text {glob }}=1.5$ and $\alpha_{\text {loc }}=1.0$, respectively.

In treated mice, a net change was observed in the slopes of the power spectra of their tumors (Fig. 1). Except in two cases, in which the tumor was suppressed due to an intense and maintained immune response, the same result was obtained. The figure shows some typical power spectra, with a characteristic global roughness exponent of $\alpha_{\mathrm{glob}}^{\mathrm{GM}-\mathrm{CSF}}=1.25 \pm 0.1$. This shows a clear change in the dynamics of tumor development. In both cases, the exponent of local roughness is the same, $\alpha_{\mathrm{loc}}^{\text {control }}=\alpha_{\mathrm{loc}}^{\mathrm{GM}-\mathrm{CSF}}=0.90 \pm 0.1$ (figure not shown).

These new dynamics are compatible with the quenched Edwards-Wilkinson (QEW) universality class, which describes the development of an elastic interface immersed in a two-dimensional random medium (modeled by a quenched noise). The equation describing its development is 

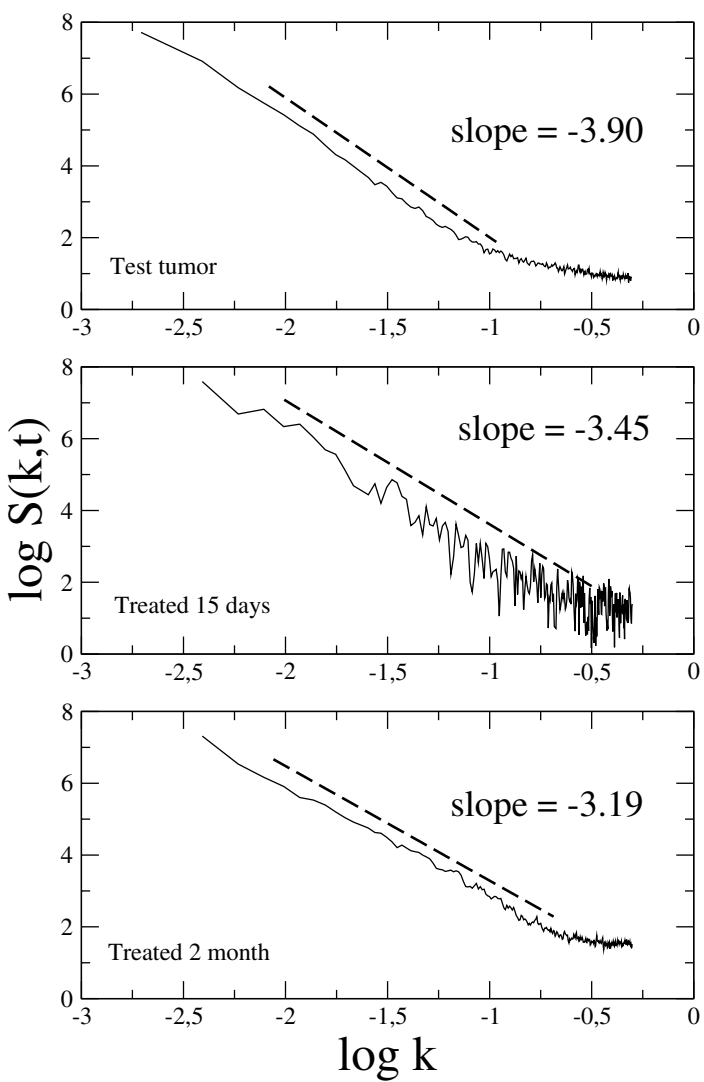

FIG. 1. Power spectra of typical tumor contours: control group (above), treated group after 15 days of treatment (middle), and treated group after two months of treatment (bottom). The corresponding fits lead to $\alpha_{\text {glob }}^{\text {control }}=3.90 \pm 0.15, \alpha_{\text {glob }}^{15 \text { days }}=3.45 \pm 0.10$, and $\alpha_{\text {glob }}^{2 \text { months }}=$ $3.19 \pm 0.05$, respectively. The nontreated group has dynamics compatible with the MBE universality class (as in any type of tumor), while those of the group treated for 15 days are compatible with the QEW universality class corresponding to pinned elastic membranes moving in a disordered medium. Finally, if treatment continues, a decrease in the slope can be observed as an effect of the neutrophils.

$$
\frac{\partial}{\partial t} h(x, t)=\frac{\partial^{2}}{\partial x^{2}} h(x, t)+F+\eta(x, h)
$$

which gives the theoretical values of $\alpha_{\text {glob }}=1.25$ and $\alpha_{\text {loc }}=0.97$ for the global and local roughness [11-13], respectively.

The first term on the right represents surface tension which favors a smooth interface; the second term is a random field which represents the two-dimensional quenched disorder (notice the dependence on $x$ and $h$ ) plus the driving force, $F$. The noise $\eta(x, h)$ has a mean of zero and short-range correlations.

These dynamics show a pinning-depinning transition $[14,15]$ characterized by a critical value of the driving force, $F_{c}$. For $F>F_{c}$, the interface moves with a nonzero velocity, whereas for $F<F_{c}$ the interface is pinned by impurities in the medium. A pinned interface typically shows large local slopes around the pinning centers.

Figure 2 shows a section of the tumor of a treated mouse. The large, darkish cells are neutrophils. The tumor interface has been marked with a red line. The pinning effect of neutrophils at the surface is obvious from their distribution. They fill concavities (they prefer regions where there are new tumor cells) all along the interface, thus inhibiting surface diffusion and increasing the pressure on the tumor cells. The immediate consequence is a decrease in the driving force, $F$. If $F$ drops below the critical value $F_{c}$ a pinning of the interface occurs, and the tumor stops growing. This kills the tumor cells causing the necrosis of around $80 \%-90 \%$ of the tumor in all cases studied.

Large necrotic areas inside tumors (with neutrophil infiltrate) are also observed by NMR after 11 weeks of therapy. Figure 3 shows a typical NMR image in which a large necrotic core (the dark region inside the tumor) can be seen. This is a direct consequence of the pressure increase induced by the pinning of the tumor interface by the neutrophils. Cells inside the tumor inhibit their proliferation as a consequence of a lack of room to do it. When cells remain quiescent for a long period of time they become necrotic $[2,16]$.

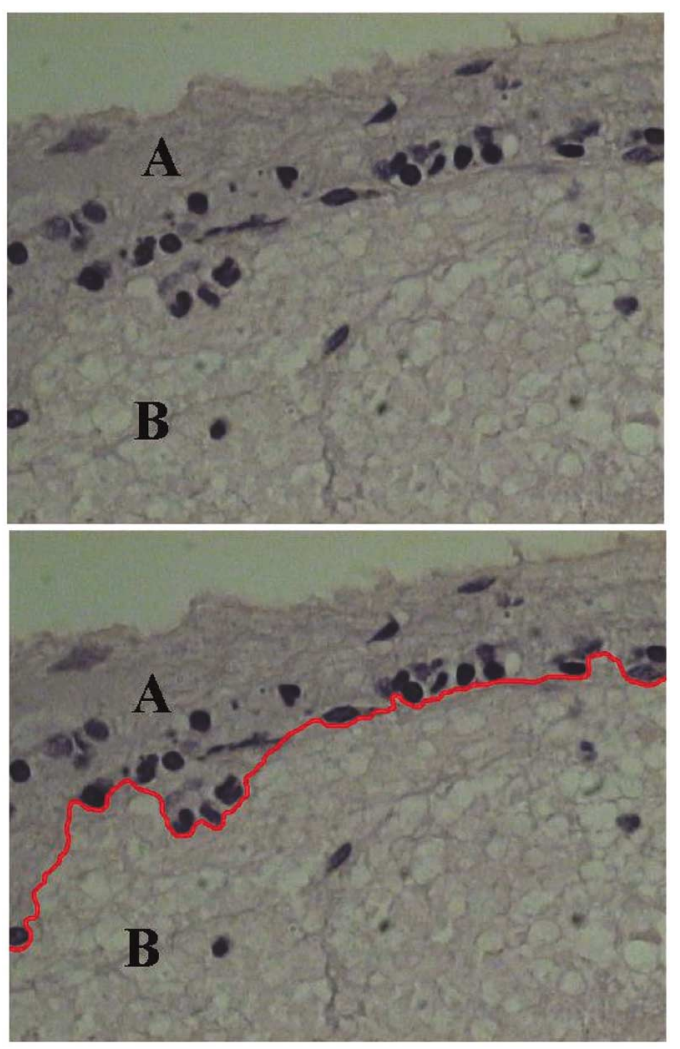

FIG. 2 (color). Region $A$ is the host and region $B$ the tumor. In the bottom picture, the host-tumor interface is highlighted in red. Note how the neutrophils locate themselves to eliminate tumor cell surface diffusion. 


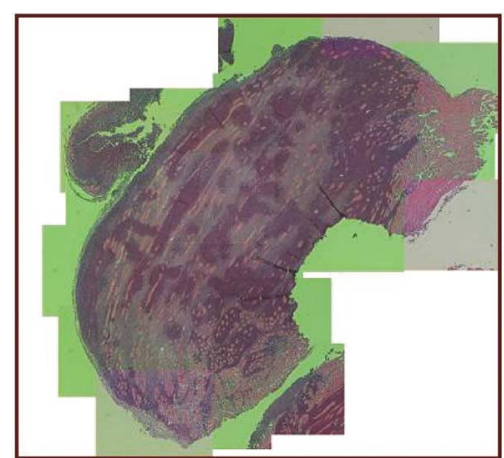

a)

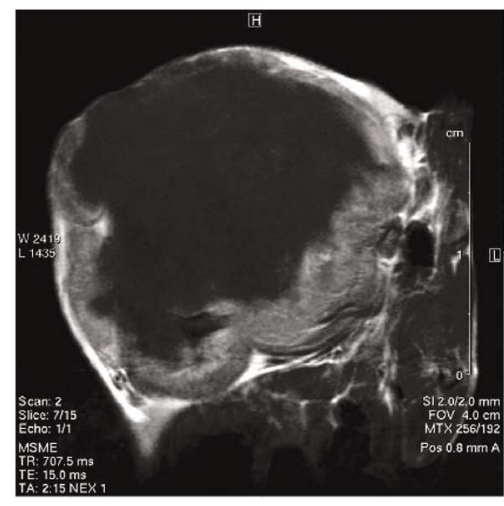

b)

FIG. 3 (color). (a) Hematoxylin-eosin composite of histological sections of a mouse tumor. The dark purple regions are proliferative regions of the tumor. (b) The necrotic regions appear darker in the NMR picture of tumor. The tumor is stained with gadolinium and appears in gray. White regions are fat. The necrotic core is black.

Treatment led to an increased immune response that was maintained over a long period. This caused the tumor dynamics to undergo a first transition from MBE to QEW. Continuation of the treatment led to these cells inducing a second transition to another set of even slower dynamics, as yet uncharacterized but with a global roughness exponent of $\alpha_{\text {glob }}^{2 \text { months }}=1.10 \pm 0.10$ and with $\alpha_{\text {loc }}^{2 \text { months }}=$ $0.90 \pm 0.10$. As a consequence of pinning at the interface, the activity of the tumor itself causes its own decline. Since the cells at the border can no longer find sufficient space, they can no longer proliferate, and those inside the tumor eventually become necrotic (as shown in Fig. 3). This is compatible with that seen in tumors growing in vitro under pressures greater than atmospheric. Such pressures exert an effect similar to that of the neutrophils [16].

The most striking clinical result of this therapy is the increase in survival time. The death ratio in the control group is 15.76 times higher than in the treated group (equivalent to a Cox's model hazard ratio of $0.85 ; 95 \%$ confidence interval $0.76-0.95, p=0.004$ ).

These experiments support the idea that tumor growth is largely governed by environmental pressures (host tissue pressure, immune response, etc.) - pressures that fundamentally influence tumor dynamics. In our previous work, we established the universality of these dynamics (i.e., for all kinds of tumors) and their corresponding to a surface growth phenomenon. By enhancing the immune response in a group of mice with implanted tumors, we have shown here, both through direct observations and through changes in the dynamics from MBE to QEW, how neutrophils pin the interface. The therapeutic effect of this change in dynamics includes extensive necrosis of the tumor-sometimes complete elimination-and an important increase in survival time. The possibilities this points to are of great importance in the treatment of cancer, especially since increasing the immune response in the way used here has very few side effects in humans [17]. These experiments also stress the importance of understanding the physics of cancer growth and the immune response, and show the latter to have a mechanical effect on the former similar to that produced by high external pressure.

We would like to thank J.A. Cuesta and M. A. Rodríguez for their helpful comments and suggestions and Jesús Pastor for his support.

\footnotetext{
*Electronic address: antonio.bru@ccma.csic.es
}

[1] A. Brú, J. M. Pastor, I. Fernaud, S. Melle, I. Brú, and C. Berenguer, Phys. Rev. Lett. 81, 4008 (1998).

[2] A. Brú, S. Albertos, J. Luis Subiza, J. L. García-Asenjo, and I. Brú, Biophys. J. 85, 2948 (2003).

[3] H. Kallabis, L. Brendel, J. Krug, and D. E. Wolf, Int. J. Mod. Phys. B 11, 3621 (1997).

[4] Z.-W. Lai and S. Das Sarma, Phys. Rev. Lett. 66, 2348 (1991).

[5] M. B. Hallet, D. Emlay, and P. D. Kramer, Neutrophil: Cellular Biochemistry and Physiology (CRC Press, Boca Raton, FL, 1989).

[6] S. Green, J. Benedetti, and J. Crowley, Clinical Trials in Oncology (Chapman and Hall, London, 1998), 3rd ed.

[7] N. E. Breslow, Biometrics 30, 89 (1974).

[8] D. R. Cox, J. R. Statist. Soc. Ser. B 34, 187 (1972).

[9] J. Villain, J. Phys. I 1, 19 (1991).

[10] L.-H. Tang and T. Nattermann, Phys. Rev. Lett. 66, 2899 (1991).

[11] M. Dong, M. C. Marchetti, A. A. Middleton, and V. Vinokur, Phys. Rev. Lett. 70, 662 (1993).

[12] H. Leschorn, Physica (Amsterdam) 195A, 324 (1993).

[13] M.-P. Kuittu, M. Haataja, and T. Ala-Nissila, Phys. Rev. E 59, 2677 (1999).

[14] H. A. Makse, S. Buldyrev, H. Leschhorn, and H. E. Stanley, Europhys. Lett. 41, 251 (1998).

[15] R. Bruinsma and G. Aeppli, Phys. Rev. Lett. 52, 1547 (1984).

[16] A. Brú, S. Albertos, J. A. López García-Asenjo, and I. Brú (to be published).

[17] M. D. Caballero et al., Haematologica 83, 514 (1998). 